\title{
Adventures in drugging undruggable targets
}

\author{
David Spring \\ University of Cambridge, United Kingdom
}

https://doi.org/10.17952/35EPS.2018.008

Pharmaceutical chemistry research has traditionally focused upon a limited set of biological targets. Many other human disease-related targets have been termed 'undruggable' as they have proved largely impervious to modulation by small molecules. However, it is becoming increasingly evident that such targets can indeed be modulated; they are simply being challenged with the wrong types of molecules. Research using conformationally constrained peptides should provide hits against a broad range of targets with high frequency, including 'undruggable' targets.

Protein-protein interactions (PPIs) have emerged as attractive drug discovery targets in recent years due to their key roles in mediating various cellular functions. PPIs are however notoriously challenging to target; therefore, they have been termed 'undruggable'. The interfaces that characterize PPIs are often large, shallow, and highly flexible, making rational drug design difficult. Nevertheless, there is growing interest in exploring these, particularly through harnessing peptides as PPI-targeting drugs due to their potency, high specificity, and low toxicity. Peptides are also viewed as a bridging class of drugs that could potentially combine the desirable properties of small molecules with those of biologics. Despite numeroussuccessful examples of peptide-based drugs that have already reached the market, peptides are often considered poor drug candidates because of their low bioavailability, rapid elimination, poor in vivo stability, and administration. These limitations are now gradually being mitigated by advances in peptide delivery and synthetic methodologies. An importantadvance in the development of peptide-based drugs is the introductionof the "stapling" strategy.

Incorporation of staples (conformational constraints) within the peptide has been extensively used to improve the bioavailability of these molecules; consequently, it is not surprising that a plethora of stapling techniques has been developed and has had a significant impact on the development of peptide therapeutics. A mong the numerousstapling techniques known, two-componentmethodologies allow facile and divergent functionalization of peptides. The author's laboratory has pioneered a stapling technique that makes use of the double Cu-catalyzed azide-alkyne cycloaddition between di-azido peptides and functionalized di-alkynyl staples. In recent years, the author's laboratory has created biologically active, conformationally constrained peptides functionalized with cell-penetrating peptides, fluorescent tags, and photo cross-linking moieties, demonstrating the wide applicability of this methodology. The impact, advantages, limitations, and future applications of this technology and other two-component peptide stapling techniques on the development of clinically relevant peptides were highlighted.

Examples in the area of two-component peptide stapling methodology taken from the author's laboratory included:

•p53-MDM2 Interaction[1,2,3,4,5]

-Targeting the Genome-Stability Hub Ctf4[6]

•HNF1 $\beta$ - Importin $\alpha$ PPI[7]

-Tankyrase - Axin PPI[8]

Peptide therapeutics represent an emerging area in the pharmaceutical field. Considering their selectivity, efficacy, low toxicity, and cost of goods, peptides are the ideal molecules to target shallow areas of proteins, such as the interface of PPIs, thus unlocking a large number of extra and intracellular pathways that can be disrupted. The main drawbacks of this class of molecules are their inherent instability in vivo and their weak bioavailability. Significant progress has been made in increasing the resistance of the peptide to proteases and improving their cell-permeability and hence, tissue penetration. Macrocyclization of linear peptides is certainly one of the most successful methodologies to overcome many of the limitations of peptides, and a plethora of different techniques have been developed to this end. In this lecture, we have highlighted the recent advances made in peptide stapling, the advantage of the two-component peptide stapling techniques over one-component and, in particular, the achievements obtained with the rational design of two-component $\mathrm{Cu}$-catalyzed azide-alkyne 
cycloaddition stapled peptides. The two-component peptide stapling methodologies involve an intramolecular reaction between the two amino acids on the linear peptide and a staple or linker that can bear a functional handle for further functionalization. After merging the two components together, the peptide may be constrained in the bioactive conformation minimizing the entropic penalty upon binding of the peptide to the targeted protein and increasing its stability to enzymatic degradation. This methodology has been successfully applied not only to constrain $\alpha$-helical peptides, but also to random coils, therefore expanding its scope. Using the $\mathrm{Cu}-$ catalyzed azide-alkyne cycloaddition two-component peptide stapling technique developed in our group, we have created a toolbox of staples not only suitable for constraining different secondary structures of selected sequences, but also stapling at different positions and conditions while also bearing different functionalities. In addition, our two-component peptide stapling methodologies have been proven to be compatible with all the biogenic amino acids that may be present. The toolbox includes staples carrying fluorescent tags, cellpermeable motifs, biotin tags, nuclear localization sequences, and photoaffinity labelling moieties, showcasing the wide applicability of this system. Moreover, stapled peptides obtained were often found to be more active against the set target than the unstapled variants and wild-type peptides. Most importantly, they showed improved cell-permeability and in vitro stability. Encouraged by the positive results obtained so far, we envisioned that the scope of the two-component peptide stapling can be expanded further by adding more functionalized stapled peptides to the toolbox.

\section{References}

1. Y. H. Lau, P. de Andrade, S.-T. Quah, M. Rossmann, L. Laraia, N. Sköld, T. J. Sum, P. J. E. Rowling, T. L. Joseph, C. Verma, M. Hyvönen, L. S. Itzhaki, A. R. Venkitaraman, C. J. Brown, D. P. Lane, D. R. Spring. Functionalised staple linkages for modulating the cellular activity of stapled peptides. Chem. Sci. 2014, 5, 1804-1809. DOI: $10.1039 / C 4 S C 00045 E$

2. Y. H. Lau, P. de Andrade, N. Sköld, G. J. McKenzie, A. R. Venkitaraman, C. Verma, D. P. Lane, D. R. Spring. Investigating peptide sequence variations for 'double-click' stapled p53 peptides. Org. Biomol. Chem. 2014, 12, 4074-4077. DOI:10.1039/C4OB00742E

3. Y. H. Lau, P. de Andrade, G. J. McKenzie, A. R. Venkitaraman, D. R. Spring. Linear aliphatic dialkynes as alternative linkers for double-click stapling of p53-derived peptides. Chembiochem 2014, 15, 2680-2683. DOI: $10.1002 /$ cbic. 201402374

4. Y. H. Lau, Y. Wu, P. de Andrade, W. R. J. D. Galloway, D. R. Spring. A two-component'double-click' approach to peptide stapling. Nature Protoc. 2015, 10, 585-594. DOI: 10.1038/nprot.2015.033

5. Y. H. Lau, Y. Wu, M. Rossmann, B. X. Tan, P. de Andrade, Y. S. Tan, C. Verma, G. J. McKenzie, A. R. Venkitaraman, M. Hyvönen, D. R. Spring. Double Strain-Promoted Macrocyclization for the Rapid Selection of Cell-Active Stapled Peptides. Angew. Chem. Int. Ed. 2015, 54, 15410-15413. DOI: 10.1002/anie.201508416

6. Y. Wu, F. Villa, J. Maman, Y. H. Lau, L. Dobnikar, A. Simon, K. Labib, D. R. Spring, L. Pellegrini. Targeting the genome stability hub Ctf4 by stapled-peptide design. Angew. Chem. Int. Ed. 2017, 56, 12866-12872. DOI: 10.1002/anie. 201705611

7. Target a Key Protein-ProteinInteraction in Ovarian Cancer. Angew. Chem. Int. Ed. 2017, 56, 524-529. DOI: 10.1002/anie.201609427

8. W. Xu, Y. H. Lau, G. Fischer, Y. S. Tan, A. Chattopadhyay, M. de la Roche, M. Hyvönen, C. Verma, D. R. Spring, L. S. Itzhaki. Macrocyclised extended peptides: Inhibiting the substrate-recognition domain of tankyrase. J. Am. Chem. Soc. 2017, 139, 2245-2256. DOI: 10.1021/jacs.6b10234 